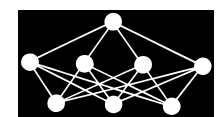

\title{
CONNECTIVITY OF INFERIOR CEREBELLAR PEDUNCLE IN THE HUMAN BRAIN: A DIFFUSION TENSOR IMAGING STUDY
}

\author{
S.H. Jang, H.G. Kwon
}

\begin{abstract}
The inferior cerebellar peduncle (ICP) is an important role in motor control, such as coordination of movement control of balance, posture, and gait. In the current study, using diffusion tensor tractography (DTT), we attempted to investigate the connectivity of the ICP in normal subjects. Forty healthy subjects were recruited for this study. DTTs were acquired using a sensitivity-encoding head coil at 1.5 Tesla. A seed region of interest was drawn at the ICP using the FMRIB Software Library. Connectivity was defined as the incidence of connection between the ICP and target brain regions at the threshold of 5, 25, and 50 streamlines. The ICP showed $100 \%$ connectivity to the vestibular nucleus, reticular formation, pontine tegmentum, and posterior lobe of the cerebellum, irrespective of thresholds. In contrast, the ICP showed more than $70 \%$ connectivity with the target brain regions at the threshold of 5 streamlines that is to the thalamus $(100 \%)$, anterior lobe of the cerebellum $(100 \%)$, pedunculopontine nucleus $(95.0 \%)$, red nucleus $(92.5 \%)$, primary somatosensory cortex $(86.3 \%)$, and primary motor cortex $(75.0 \%)$. According to our findings, the ICP had high connectivity, mainly with the sensory-motor related areas. We believe that the methodology and results of this study would be useful in investigation of the neural network associated with the sensory-motor system and brain plasticity following brain injury and other diseases.
\end{abstract}

Key words: inferior cerebellar peduncle, restiform body, connectivity, cerebellum, diffusion tensor imaging

Received: February 4, 2015

DOI: $10.14311 /$ NNW.2016.26.025

Revised and accepted: September 19, 2016

\section{Introduction}

The cerebellum as a key role of motor coordination and cognitive function communicates cerebrum via cerebellar peduncles $[34,36]$. The inferior cerebellar peduncle (ICP, also called the restiform body) is located in the dorsolateral border

Sung Ho Jang, Hyeok Gyu Kwon - Corresponding author, Department of Physical Medicine and Rehabilitation, College of Medicine, Yeungnam University 317-1, Daemyung dong, Namku, Daegu, 705-717, Republic of Korea, E-mail: strokerehab@hanmail.net, khg0715@hanmail.net 
of the medulla oblongata and contains several afferent and efferent tracts (dorsal spinocerebellar tract, cuneocerebellar tract, olivocerebellar tract, reticulocerebellar tract, vestibulocerebellar tract, arcuatocerebellar tract, and trigeminocerebellar tract, cerebellovestibular tract, cerebelloreticular tract, and cerebello-olivary tract) $[1,6,26,30-32]$. It mainly conveys somatosensory information, such as proprioceptive from muscle spindles and tendons, and vestibular information from vestibular nuclei to cerebellum $[1,6,26]$. Therefore, it has an important role in motor control such as coordination of movement control of balance, posture, and gait $[6,8,13$, $23,26,31,37]$.

Thorough comprehension of the connectivity of a neural structure would be important for research on normal neurophysiology and neural plasticity, which is based on the unmasking of latent neural connection after brain injury [2, 40, 41]. However, research on the connectivity of a neural structure is difficult because neurons interact with each other in a wide and complicated neural network. On the other hand, recently developed diffusion tensor tractography (DTT), derived from diffusion tensor imaging (DTI), has an unique advantage in estimation and visualization of the connections by detection of water diffusion properties [4, 29]. In particular, the probabilistic tracking method, based on the multi-tensor model, enables calculation of more than one fiber population in each imaging voxel $[4,29$, 35]. As a result, many studies using a probabilistic tracking method have reported on connectivity of neural structures in the live human brain $[11,14,16,17,22$, $28,29]$ and regarding the ICP, many studies have reported on the ICP in terms of tract (or pathway) related to the cerebellum using deterministic tracking method which can trace the only dominant orientation of diffusion in each voxel $[7,9,18$, 21]. However, little is known about connectivity of the ICP in the normal human brain.

In the current study, using probabilistic tracking DTT, we attempted to investigate the ipsilateral connectivity of the ICP in normal subjects.

\section{Methods}

\section{$2.1 \quad$ Subjects}

We recruited 40 healthy subjects (males: 24, females: 16, mean age: 32.4 years, range: $22 \tilde{5} 0$ years) with no previous history of neurological, physical, psychiatric illness or head trauma. No abnormal lesion in all subjects was observed in brain MRI by a neuroradiologist. All subjects understood the purpose of the study and provided written, informed consent prior to participation. The study protocol was approved by our local Institutional Review Board.

\subsection{Data acquisition}

A 6-channel head coil on a 1.5T Philips Gyroscan Intera (Philips, Ltd, Best, The Netherlands) with single-shot echo-planar imaging (EPI) was used for acquisition of DTI data. For each of the 32 non-collinear, diffusion-sensitizing gradients, we acquired 67 contiguous slices parallel to the anterior commissure-posterior commissure line. Imaging parameters were as follows: acquisition matrix $=96 \times 96$; 
reconstructed to matrix $=128 \times 128$; field of view $=221 \times 221 \mathrm{~mm}$; repetition time $(\mathrm{TR})=10,726 \mathrm{~ms}$; echo time $(\mathrm{TE})=76 \mathrm{~ms}$; parallel imaging reduction factor $($ SENSE factor $)=2$; echo-planar imaging $(\mathrm{EPI})$ factor $=49 ; b=1000 \mathrm{~s} / \mathrm{mm}^{2}$; number of excitations $(\mathrm{NEX})=1$; and a slice thickness of $2.3 \mathrm{~mm}$ (acquired voxel size $1.73 \times 1.73 \times 2.3 \mathrm{~mm})$.

\subsection{Probabilistic fiber tracking}

Analysis of DTI data was performed using the Oxford Centre for Functional Magnetic Resonance Imaging of the Brain (FMRIB) Software Library (FSL; www. fmrib.ox.ac.uk/fsl). Head motion effect and image distortion due to eddy current were corrected by affine multi-scale two-dimensional registration. Fiber tracking was performed using a probabilistic tractography method based on a multi-fiber model, and applied in the current study utilizing tractography routines implemented in FMRIB Diffusion (5000 streamline samples, $0.5 \mathrm{~mm}$ step lengths, curvature thresholds $=0.2)[4,35]$. For the connectivity of the ICP, a seed region of interest was drawn at the isolated ICP area of the medulla on the axial image $[12,27,32]$. The ICP was identified by reconstructing adjacent structures, that is, the spinothalamic tract (anterior boundary) and the corticoreticular tract (medial boundary). Out of 5000 samples generated from a seed voxel, results were visualized at the threshold of 5, 25, and 50 streamlines through each voxel for analysis (Fig. 1).

\subsection{Determination of connections between the ICP and target brain regions}

Connectivity was defined as the incidence of connection between the ICP and each brain region: primary motor cortex (M1, brodmann area [BA]: 4), primary somatosensory cortex (S1, BA: 1, 2, 3), premotor cortex (PMC, BA: 6), prefrontal cortex (BA: 9, 10, 11, 12), globus pallidus, thalamus, red nucleus, decussation portion of midbrain, pedunculopontine nucleus ( $\mathrm{PPN})$, pontine tegmentum, vestibular nucleus, reticular formation, anterior lobe of the cerebellum, posterior lobe of the cerebellum, and flocculonodular lobe of the cerebellum [5]. Incidence of the connectivity was counted the connections from the ICP to each brain region and then the incidence of connectivity calculated as the percentage in all hemispheres of 40 subjects.

\subsection{Statistical analysis}

SPSS software (v.15.0; SPSS, Chicago, IL) was used for the analysis. The chi-square test was used for determination of the incidences of connectivity and differences between incidences of connectivity of the ICP in the right and left hemispheres in each subject and between males and females. Statistical significance was accepted for $p$ values of $<0.05$. 


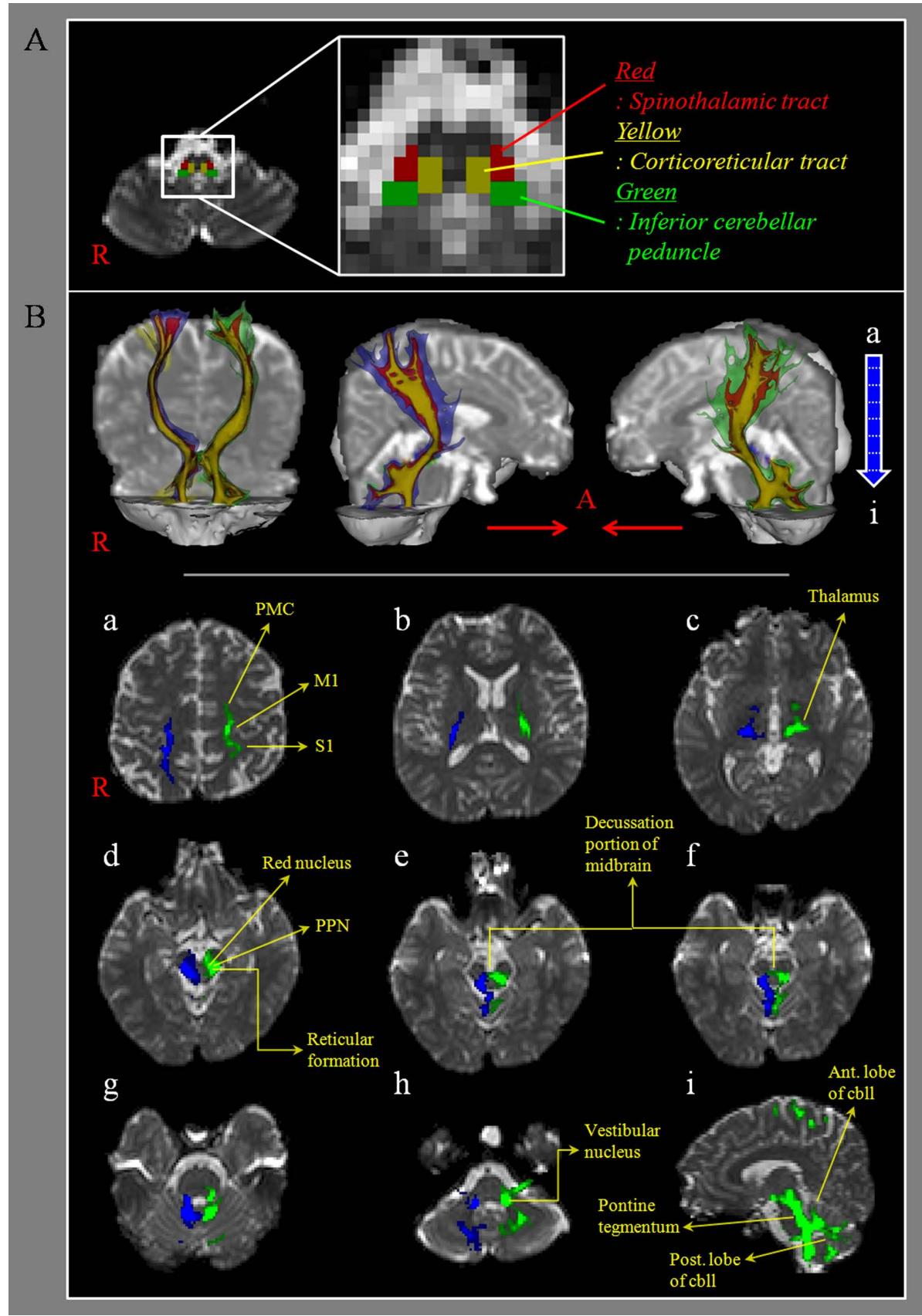

Fig. 1 Results of diffusion tensor tractography for the connectivity between the ICP and target brain regions at a threshold of 5 (right: blue left: green), 25 (red), and 50 (yellow). (a: cortex level, b: corona radiata level, c: internal capsule level, $d$ : anterior commissural level, e: upper midbrain level, f: middle midbrain level, g: lower midbrain level, $h$ : upper pons level, $i$ : lower pons level). 


\section{Results}

A summary of the connectivity of the ICP is shown in Tab. I. The ICP showed $100 \%$ connectivity with the vestibular nucleus, reticular formation, pontine tegmentum, and posterior lobe of the cerebellum, irrespective of thresholds. In contrast, regarding the threshold of 5, 25, and 50 streamlines, the ICP showed connectivity to the thalamus $(100 \%, 96.3 \%$, and $96.3 \%)$, anterior lobe of the cerebellum $(100 \%$, $92.5 \%$, and $92.5 \%)$, PPN $(95.0 \%, 91.3 \%$, and $91.3 \%)$, red nucleus $(92.5 \%, 83.8 \%$, and $78.8 \%$ ), S1 $(86.3 \%, 70.0 \%$, and $58.8 \%)$, M1 $(75.0 \%, 38.8 \%$, and $32.5 \%)$, decussation portion of midbrain $(66.3 \%, 56.3 \%$, and $53.8 \%)$, globus pallidus $(53.8 \%$, $40.0 \%$, and $31.3 \%$ ), flocculonodular lobe of the cerebellum $(32.5 \%, 25.0 \%$, and $13.8 \%)$, prefrontal cortex $(15.0 \%, 8.8 \%$, and $6.3 \%)$, and PMC $(11.3 \%, 5.0 \%$, and $5.0 \%$ ), respectively. According to statistical analysis, no significant differences in connectivity of the ICP were observed between the right and left hemispheres in each subject and between males and females $(p>0.05)$.

\begin{tabular}{lccc}
\hline \multirow{2}{*}{ Target brain regions } & \multicolumn{3}{c}{ Threshold } \\
& $5[\%]$ & $25[\%]$ & $50[\%]$ \\
\hline Vestibular nucleus & 100 & 100 & 100 \\
Reticular formation & 100 & 100 & 100 \\
Pontine tegmentum & 100 & 100 & 100 \\
Posterior lobe of cerebellum & 100 & 100 & 100 \\
Thalamus & 100 & 96.3 & 96.3 \\
Anterior lobe of cerebellum & 100 & 92.5 & 92.5 \\
Pedunculopontine nucleus & 95.0 & 91.3 & 91.3 \\
Red nucleus & 92.5 & 83.8 & 78.8 \\
Primary somatosensory cortex & 86.3 & 70.0 & 58.8 \\
Primary motor cortex & 75.0 & 38.8 & 32.5 \\
Decussation portion of midbrain & 66.3 & 56.3 & 53.8 \\
Globus pallidus & 53.8 & 40.0 & 31.3 \\
Flocculonodular lobe of cerebellum & 32.5 & 25.0 & 13.8 \\
Prefrontal cortex & 15.0 & 8.8 & 6.3 \\
Premotor cortex & 11.3 & 5.0 & 5.0 \\
\hline
\end{tabular}

Tab. I Incidence of connectivity [\%] between the inferior cerebellar peduncle and target brain regions.

\section{Discussion}

In the current study, using probabilistic tracking DTT, we investigated the connectivity of the ICP in the normal human brain. Our findings showed the following characteristics of connectivity of the ICP: 1) all subjects showed $100 \%$ connectivity with the vestibular nucleus, reticular formation, pontine tegmentum, and posterior lobe of the cerebellum, irrespective of thresholds, 2) the ICP showed more than $70 \%$ connectivity with the sensory-motor function related areas at the threshold 
of 5 streamline, that is, the thalamus, posterior lobe of the cerebellum, PPN, red nucleus, S1, and M1.

Many previous studies have reported on the anatomical characteristics of the ICP: the ICP connects the spinal cord and cerebellum and this connection is essential to coordinated movements $[1,6,8,26,30,31,33,37]$. In detail, the ICP receives and conveys proprioceptive information from the vestibular nucleus and reticular formation to the cerebellum. By contrast, little is known about the connectivity of the ICP in the human brain. Since the introduction of DTI, to the best of our knowledge, two studies have reported on the reconstruction of ICP or the neural tract which is relevant with ICP $[12,15]$. In 2007, Habas and Cabanis reported that the pathway of the ICP ascended and passed through the area between the lateral wall of the fourth ventricle and the middle cerebellar peduncle and then reached the vermis and paravermis of the anterior lobe of the cerebellum in the human brain [12]. In a recent study, using DTT, Jang and Kwon identified the dorsal spinocerebellar tract, which passed through the ICP in 26 normal human subjects [15]. We reported that the dorsal spinocerebellar tract showed $100 \%$ connectivity with the anterior lobe of the cerebellum. Consequently, our results appear to coincide with those of previous studies $[12,15]$. On the other hand, although the olivocerebellar tract, which conveys information from the olivary nuclei to the cerebellum, is a major tract of the ICP, we could not find any connectivity to the olivary nuclei, which might be ascribed to the kissing effect, because of the pathway of olivocerebellar tract that originated in the contralateral olivary nucleus, crossed the midline, and then terminated in the cerebellum [3, 19, 26, 31, 32].

Regarding the connectivity with the sensory-motor related areas (thalamus, posterior lobe of the cerebellum, PPN, red nucleus, S1, and M1), previous studies have reported that injury of the ICP could affect motor function with clinical manifestations such as ataxia, hypotonia, and gait disturbance $[6,8,13,23,26,31,37]$. In 2009, using DTT, Hong et al. recruited six patients with ataxia and demonstrated injury of cerebellar peduncles, including the ICP [13]. Subsequently, Kwon and Jang [2012] reported on a patient who showed hand tremor, gait disturbance, cognitive dysfunction, and truncal ataxia due to injury of the fornix, corticospinal tract, and ICP on DTT following traumatic brain injury [23]. Therefore, in terms of function of the ICP related to the sensory-motor area, our results appear to be compatible with those of previous studies [13, 23].

In conclusion, we found that the ICP has high connectivity, mainly with the sensory-motor related areas, including the vestibular nucleus, reticular formation, pontine tegmentum, thalamus, PPN, red nucleus, primary sensori-motor cortex, and cerebellum. To the best of our knowledge, this is the first study using probabilistic DTI to demonstrate the connectivity of the ICP to each brain region in the human brain. We believe that the results and methodology of this study would be useful in investigation of the neural network associated with the sensory-motor system and brain plasticity following brain injury and other diseases [7, 20, 24]. However, several limitations of this study should be considered [10, 19, 25, 29, 38, 39]. First, due to kissing or partial volume effect, DTI could lead to both false positive and negative results throughout the white matter of the brain [10, 25, 39]. In particular, probabilistic tracking could cause a false positive fiber trajectory [10, $25,39]$. Second, we could not determine the detailed connectivity area, such as the 
cerebellar nuclei, because isolation of each cerebellar nucleus is not easy with our resolution. Third, high tesla MRI can provide better resolution and detail anatomical information. Therefore, we think that conduct of further studies to overcome these limitations should be encouraged. In addition, studies for clinical application of our results for patients following brain injury are also invited.

\section{Acknowledgement}

This work was supported by the DGIST R\&D Program of the Ministry of Science, ICT and Future Planning (16-BD-0401).

\section{References}

[1] AFIFI A.K., BERGMAN R.A. Functional neuroanatomy: text and atlas. New York: Lange Medical Books/McGraw-Hill, 2005, pp. ix, 494.

[2] BACH-Y-RITA P. Brain plasticity as a basis of the development of rehabilitation procedures for hemiplegia. Scandinavian journal of rehabilitation medicine. 1981, 13(2-3), pp. 73-83.

[3] BASSER P.J., PAJEVIC S., PIERPAOLI C., DUDA J., ALDROUBI A. In vivo fiber tractography using DT-MRI data. Magnetic resonance in medicine: official journal of the Society of Magnetic Resonance in Medicine / Society of Magnetic Resonance in Medicine. 2000, 44(4), pp. 625-632.

[4] BEHRENS T.E., BERG H.J., JBABDI S., RUSHWORTHA M.F.S., WOOLRICHA M.W. Probabilistic diffusion tractography with multiple fibre orientations: What can we gain? NeuroImage. 2007, 34(1), pp. 144-155, doi: 10.1016/j.neuroimage. 2006.09.018.

[5] BRODMANN K., GARY L.J.. Brodmann's localization in the cerebral cortex: the principles of comparative localisation in the cerebral cortex based on cytoarchitectonics. New York: Springer, 2006, pp. xv, 298.

[6] BROUSSARD D.M. The cerebellum: learning movement, language, and social skills. Singapore: WILEY Blackwell, 2014, pp. 226.

[7] CATANi M., JONES D.K., DALY E., EMBIRICOSA N., DEElEYA Q., PUGLIESEA L., CURRANA S., ROBERTSONA D., MURPHY D.G.M. Altered cerebellar feedback projections in Asperger syndrome. NeuroImage. 2008, 41(4), pp. 1184-1191, doi: 10.1016/j. neuroimage.2008.03.041.

[8] DELISA J.A. Rehabilitation medicine: principles and practice. Philadelphia: Lippincott, 1993, pp. xxiii, 1238.

[9] DRIJKOningen D., CAEYENBERGHS K., LEUNISSEN I., LINDENC C.V., LEEMANSF A., SUNAERTD S., DUYSENSA J., SWINNEN S.P. Training-induced improvements in postural control are accompanied by alterations in cerebellar white matter in brain injured patients. NeuroImage: Clinical. 2015, 7, pp. 240-251, doi: 10.1016/j.nicl.2014. 12.006 .

[10] Fillard P., DESCOTEAUX M., GOH A., GOUTTARDE S., JEURISSENF B., MALCOLMG J., RAMIREZ-MANZANARESH A., REISERTI M., SAKAIEJ K., TENSAOUTIK F., YOL T., MANGINB J.-F., POUPON C. Quantitative evaluation of 10 tractography algorithms on a realistic diffusion MR phantom. NeuroImage. 2011, 56(1), pp. 220-234, doi: $10.1016 / j$.neuroimage .2011 .01 .032 .

[11] GUYE M., PARKER G.J., SYMMS M., BOULBYA P., WHEELER-KINGSHOTTC C.A.M., SALEK-HADDADIA A., BARKERC G.J., DUNCAN J.S. Combined functional MRI and tractography to demonstrate the connectivity of the human primary motor cortex in vivo. NeuroImage. 2003, 19(4), pp. 1349-1360.

[12] HABAS C., CABANIS E.A. Anatomical parcellation of the brainstem and cerebellar white matter: a preliminary probabilistic tractography study at 3 T. Neuroradiology. 2007, 49(10), pp. 849-863, doi: 10.1007/s00234-007-0267-4. 


\section{Neural Network World 5/2016, 439-447}

[13] HONG J.H., KIM O.L., KIM S.H., LEE M.Y., JANG S.H. Cerebellar peduncle injury in patients with ataxia following diffuse axonal injury. Brain research bulletin. 2009, 80(1-2), pp. 30-35, doi: 10.1016/j.brainresbull.2009.05.021.

[14] JANG S.H., CHANG P.H., KWON H.G. The neural connectivity of the inferior olivary nucleus in the human brain: a diffusion tensor tractography study. Neuroscience letters. 2012, 523(1), pp. 67-70, doi: 10.1016/j.neulet.2012.06.043.

[15] JANG S.H., KWON H.G. Diffusion tensor tractography for the dorsal spinocerebellar tract in the human brain. Somatosensory \& motor research. 2014, 31(1), pp. 7-10, doi: 10.3109/ 08990220.2013 .813843

[16] JANG S.H., KWON H.G. Neural connectivity of the anterior body of the fornix in the human brain: diffusion tensor imaging study. Neuroscience letters. 2014, 559, pp. 72-75, doi: $10.1016 / j$.neulet. 2013.11.040.

[17] JANG S.H., KWON H.G. Neural connectivity of the posterior body of the fornix in the human brain: diffusion tensor imaging study. Neuroscience letters. 2013, 549, pp. 116-119, doi: $10.1016 / j$.neulet.2013.06.017.

[18] JANG S.H., YI J.H., KWON H.G. Injury of the inferior cerebellar peduncle in patients with mild traumatic brain injury: a diffusion tensor tractography study. Brain injury. 2016, pp. 1-5, doi: 10.1080/02699052.2016.1178805.

[19] JEURISSEN B., LEEMANS A., TOURNIER J.D., JONES D.K., SIJBERS J. Investigating the prevalence of complex fiber configurations in white matter tissue with diffusion magnetic resonance imaging. Human brain mapping. 2013, 34(11), pp. 2747-2766, doi: 10.1002/hbm. 22099.

[20] KANAAN R.A., BORGWARDT S., MCGUIRE P.K., CRAIG M.C., MURPHY D.G.M. PICCHIONI M., SHERGILL S.S., JONES D.K., CATANI M. Microstructural organization of cerebellar tracts in schizophrenia. Biological psychiatry. 2009, 66(11), pp. 1067-1069, doi: $10.1016 / \mathrm{j}$. biopsych.2009.07.028.

[21] KESER Z., HASAN K.M., MWANGI B.I., KAMALI A., UCISIK-KESER F.E., RIASCOS R.F., YOZBATIRAN N., FRANCISCO N.E., NARAYANA P.A. Diffusion tensor imaging of the human cerebellar pathways and their interplay with cerebral macrostructure. Frontiers in neuroanatomy. 2015, 9, pp. 41, doi: 10.3389/fnana.2015.00041.

[22] KWON H.G., JANG S.H. Differences in neural connectivity between the substantia nigra and ventral tegmental area in the human brain. Frontiers in human neuroscience. 2014, 8, pp. 41, doi: 10.3389/fnhum. 2014.00041.

[23] KWON H.G., JANG S.H. The usefulness of diffusion tensor imaging in detection of diffuse axonal injury in a patient with head trauma. Neural Regeneration Research. 2012, 7(6), pp. 475-478, doi: 10.3969/j.issn.1673-5374.2012.06.012.

[24] LEE A.Y., JANG S.H., YEO S.S., LEE E., CHO Y.W., SON S.M. Changes in a cerebellar peduncle lesion in a patient with Dandy-Walker malformation: A diffusion tensor imaging study. Neural Regen Res. 2013, 8(5), pp. 474-478, doi: 10.3969/j.issn.1673-5374.2013. 05.012 .

[25] LEE S.K., KIM D.I., KIM J., KIM D.J., KIM H.D., KIM D.S., MORI S. Diffusion-tensor MR imaging and fiber tractography: a new method of describing aberrant fiber connections in developmental CNS anomalies. Radiographics. 2005, 25(1), pp. 53-65; doi: 10.1148/rg. 251045085

[26] MENDOZA J.E., FOUNDAS A.L. Clinical neuroanatomy: a neurobehavioral approach. New York: Springer, 2007, pp. ix, 704.

[27] MORI S., WAKANA S., NAGAE-POETSCHER L.M., VAN ZIJL P.C.M. MRI atlas of human white matter. Amsterdam: Elsevier, 2005.

[28] MUTHUSAMY K.A., SURG M., ARAVAMUTHAN B.R., KRINGELBACH M.L., JENKINSON N., VOETS N.L., JOHANSEN-BERG H., STEIN J.F., AZIZ T.Z. Connectivity of the human pedunculopontine nucleus region and diffusion tensor imaging in surgical targeting. Journal of neurosurgery. 2007, 107(4), pp. 814-820, doi: 10.3171/JNS-07/10/0814. 
Jang S.H., Kwon H.G.: Neural connectivity of inferior cerebellar peduncle

[29] PARKER G.J., ALEXANDER D.C. Probabilistic anatomical connectivity derived from the microscopic persistent angular structure of cerebral tissue. Philosophical transactions of the Royal Society of London. Series B, Biological sciences. 2005, 360(1457), pp. 893-902, doi: 10. 1098/rstb.2005.1639.

[30] PERRINI P., TIEZZI G., CASTAGNA M., VANNOZZI R. Three-dimensional microsurgical anatomy of cerebellar peduncles. Neurosurgical review. 2013, 36(2), pp. 215-224, doi: 10. 1007/s10143-012-0417-y.

[31] PRITCHARD T.C., ALLOWAY K.D. Medical neuroscience. Madison, Connecticut: Fence Creek Publishing, 1999, pp. xiii, 448.

[32] Salamon N., SiCotTe N., Alger J., Shattuck D., PERlman S., Sinha U. SCHULTZE-HAAKH H., SALAMON G. Analysis of the brain-stem white-matter tracts with diffusion tensor imaging. Neuroradiology. 2005, 47(12), pp. 895-902, doi: 10.1007/ s00234-005-1439-8.

[33] SAlAmOn N., SICOTTE N., DRAin A., FREW A., ALGER J.R., JEN J., PERLMAN S., SALAMON G. White matter fiber tractography and color mapping of the normal human cerebellum with diffusion tensor imaging. Journal of neuroradiology. 2007, 34(2), pp. 115128, doi: $10.1016 / j$.neurad.2007.03.002.

[34] SCHMAHMANN J.D. An emerging concept. The cerebellar contribution to higher function. Archives of neurology. 1991, 48(11), pp. 1178-1187.

[35] SMITH S.M., JENKINSON M., WOOLRICH M.W., BECKMANNA C.F., BEHRENSA T.E.J., JOHANSEN-BERGA H., BANNISTERA P.R., DE LUCAA M., DROBNJAKA I., FLITNEYA D.E., NIAZYA R.K., SAUNDERSA J., VICKERSA J., ZHANGA Y., DE STEFANOB N., BRADYC J.M., MATTHEWSA P.M. Advances in functional and structural MR image analysis and implementation as FSL. NeuroImage. 2004, 23, Suppl 1, pp. S208-S219, doi: $10.1016 / j$. neuroimage .2004 .07 .051 .

[36] STOODLEY C.J., SCHMAHMANN J.D. Functional topography in the human cerebellum: a meta-analysis of neuroimaging studies. NeuroImage. 2009, 44(2), pp. 489-501, doi: 10. $1016 / j$.neuroimage .2008 .08 .039 .

[37] THOMSEN I.V. Late outcome of very severe blunt head trauma: a 10-15 year second followup. Journal of neurology, neurosurgery, and psychiatry. 1984, 47(3), pp. 260-268.

[38] YAMADA K. Diffusion tensor tractography should be used with caution. Proceedings of the National Academy of Sciences of the United States of America. 2009, 106(7), pp. E14, doi: 10.1073 pnas. 0812352106.

[39] K. Yamada, K. Sakai, K. Akazawa, S. Yuen, T. Nishimura. MR tractography: a review of its clinical applications. Magnetic resonance in medical sciences. 2009, 8(4), pp. 165-174.

[40] YEO S.S., JANG S.H. Neural reorganization following bilateral injury of the fornix crus in a patient with traumatic brain injury. Journal of Rehabilitation Medicine. 2013, 45(6), pp. 595-598, doi: 10.2340/16501977-1145.

[41] YEO S.S., JANG S.H. Recovery of an injured fornix in a stroke patient. Journal of Rehabilitation Medicine. 2013, 45(10), pp. 1078-1080, doi: 10.2340/16501977-1232. 